\title{
Simultaneous spectroscopic and temperature imaging by IR monochromator and camera
}

\author{
By M. Romano*, C. Pradere*, C. Ndiaye, A.Duphil and J.C. Batsale*
}

* I2M, Departement TREFLE, UMR CNRS 5295 - site ENSAM Esplanade des Arts et Métiers, 33405 Talence

Cedex, France, romano@enscbp.fr , c.pradere@i2m.u-bordeaux1.fr, and jcbatsale@i2m.u-bordeaux1.fr

\begin{abstract}
In this paper the simultaneous measurement of IR spectroscopic imaging and temperature fields are proposed. First the experimental set-up based on a multispectral monochromator in the IR domain and the methodology will be presented. In this study, the absorbance characterization of the camera detector is shown. Then, by coupling such spectral source with a classical IR camera, images of both the temperature in proper emission and spectroscopy in transmission are presented. The preliminary results show that there is no obstacle to measure simultaneously the temperature and concentration fields as function of time. Then, with this tools it becomes possible to envisaged both thermal analysis (thermophysical properties estimation) and chemical analysis (kinetic) of chemical or phase change processes.
\end{abstract}

\section{Introduction}

A multispectral imaging spectrometer is an instrument that can simultaneously record spectral, spatial and temporal information of a sample, by studying the intensity variation of the signal as function of the time due to molecular vibrations. In fact objects are composed of vibrating atoms, and some of these atoms have higher energy and vibrates more frequently. The vibration of all charged particles and atoms generates electromagnetic waves. When the temperature of an object increases, the vibration of the atoms gets faster, and thus the spectral radiant energy rises. As a result, all objects emitted radiation at a rate function of the wavelength distribution in accordance with the temperature of the object, its spectral emissivity and chemical nature.

This kind of information is of particular importance because chemical and thermal properties of materials are strongly coupled in many applications. Chemical characterization is typically achieved by using analytical spectroscopy methods. Most of these techniques have proved to be well adapted for quantitative measurements; also they operate by scanning the surface of the sample to achieve the characterization. As a consequence, considerable efforts have been made in the past few years to develop multispectral imaging instruments [1-4]. The main purpose remain to be able to read the chemical contents on each pixel of the images through the visualization of the sample composition.

In particular, InfraRed (IR) spectroscopy has characteristics advantages, such as: fast (one minute or less per sample), non-destructive, non-intrusive, high penetration of the probing radiation beam, suitable for on-line use, and almost with nearly universal applications (any molecule containing $\mathrm{C}-\mathrm{H}, \mathrm{NH}, \mathrm{S}-\mathrm{H}$ or $\mathrm{O}-\mathrm{H}$ bonds). The combination of these characteristics with instrumental control and data treatment enhance the domain of Infrared Technology. [5-6]. Many studies regarding the evaluation of the near-infrared (NIR) spectroscopic imaging as a tool have been published: pharmaceutical applications [7] food [8], polymers [9] characterization or even to quantify the hydrated silica on Mars [10].

In this paper an IR broadband monochromator (from 1 to $21 \mu \mathrm{m}$ ) and MWIR InSb infrared camera (from 1 to $5 \mu \mathrm{m})$ will be use. First the experimental set-up, the measurement methods and the data treatment will be presented. Then, experimental results consisted in the characterization of the infrared detector overall the wavelength is done. Finally, the first results of the simultaneous measurement of the temperature and spectroscopy information during a heating process of electrical resistance is proposed. Further, with this original Infrared technology allowing to simultaneously measure the infrared spectra and temperature fields in the Middle Wave InfraRed domain (MWIR from 2$5 \mu \mathrm{m}$ ) approaches on chemical reactions or physical processes can be engaged.

\section{Experimental set-up}

The multispectral imaging instrument used in this study is sketched on figure 1. It is composed by a Bentham spectrometer for the sample analysis, which is equipped with a multispectral lamp (Nernst and halogen) emitting from $400 \mathrm{~nm}$ to $20 \mu \mathrm{m}$ and three gratings mounted on a rotated turret to mechanically select the wavelength. In this application, a grating of 150 slits is used to select a broad infrared band going from 2 to $5 \mu \mathrm{m}$ by $1 \mathrm{~nm}$ step. The diffracted light is transmitted through the sample and recorded by an infrared camera FLIR, SC7000, In-Sb focal plane array of detectors $(2-5.2 \mu \mathrm{m}, 240 \times 320$ pixels, pitch $30 \mu \mathrm{m}$ ) with a $25 \mathrm{~mm}$ objective MWIR F/2 (space resolution about $200 \mu \mathrm{m}$ ) used as sensor. In transmission mode, the camera is placed behind the sample. The sample can be thermally regulated (with PID system) by Peltier modules from $-5{ }^{\circ} \mathrm{C}$ to $70{ }^{\circ} \mathrm{C}$ for an accurate and under control cooling and heating. 


\section{Frequency synchronization}



Fig. 1. Scheme of the experimental set-up

The acquisition frequency of the IR camera was synchronized with the mechanical chopper of the spectrometer (figure 2). This configuration enables to record two different images: one with the thermal and spectroscopic information (when the chopper is open) and other containing only the thermal information (when the chopper is close). Both images were grabbed and transferred to a computer by a frame grabber. Homemade software written in Matlab language was used to control the frequency, but also to scan the wavelength domain of interest. The same software also facilitated the frame grabbing and saving data in appropriate formats for subsequent analysis based on a two images methodology.

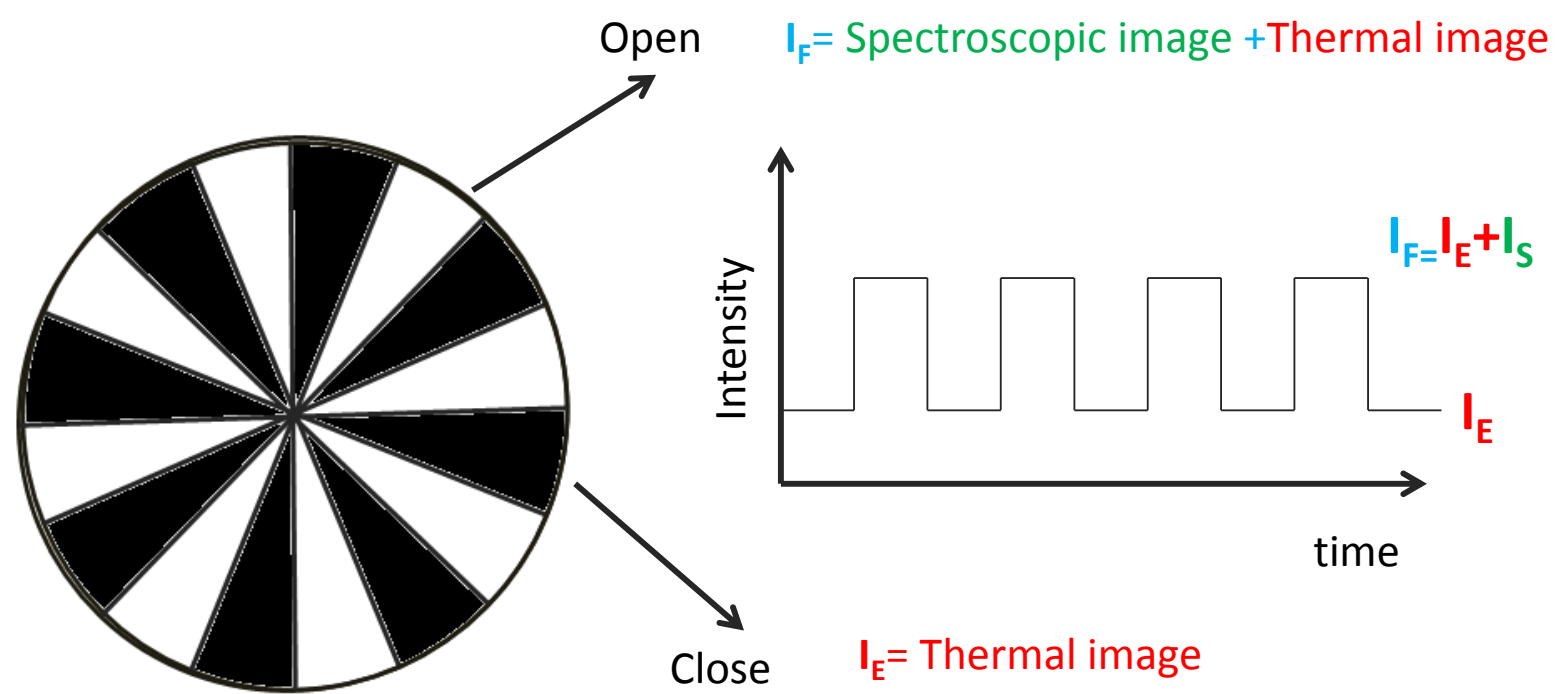

Fig. 2. Mechanical chopper placed inside the spectrometer is used to achieve the acquisition of two different images. When the chopper is close the light source from the spectrometer is cut and the recorded images of the sample contain only the thermal information (proper emission of the thermal scene) measured by the detector of the camera $\left(I_{E}\right)$. When the chopper is open the light source from the spectrometer is transmitted through the sample to the detector of the IR camera, so the recorded images $\left(I_{F}\right)$ contain the thermal information $\left(I_{E}\right)$ plus the spectrometer one $\left(I_{S}\right)$. 


\section{Experimental results}

\subsection{Characterization of the infrared sensor}

First, to calibrate the camera sensitivity overall his spectral range, a first measurement of the incident multispectral wavelength of the spectrometer was performed figure 3 . It is important to notice that the camera intensity level is strongly dependant of both the integration time and the frequency acquisition. For this raison, the measured spectrum is normalized by the maximum value overall the range as well as the subtraction of the initial baseline due to the surrounding temperature.

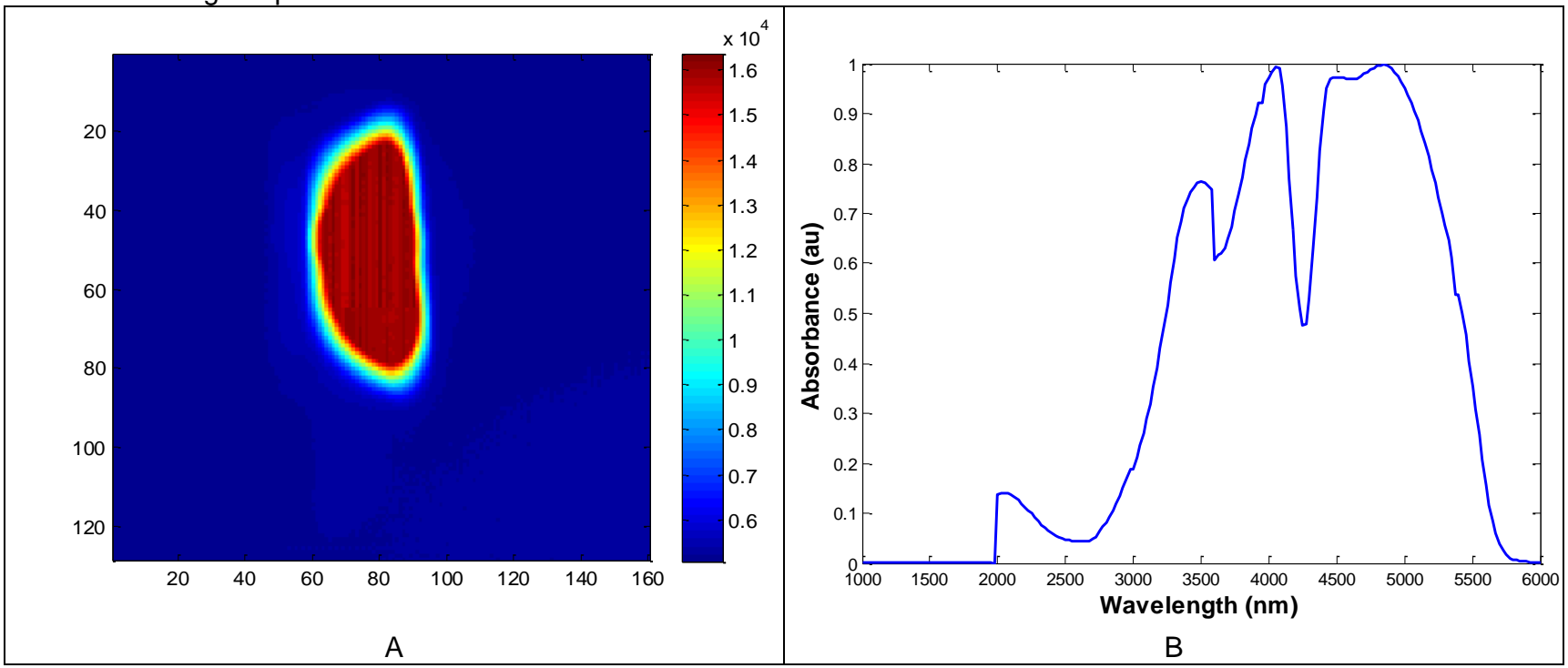

Fig. 3. Absorbance of the FLIR SC 7000 MWIR InSb camera detector: a), image of the spectrometric beam at $4 \mu \mathrm{m}$ and $\mathrm{b})$, average of the beam as function of the wavelength

\subsection{Simultaneous measurement of temperature and spectroscopy}

The total radiation received from any object is the sum of the emitted, reflected and transmitted radiation. Objects that are not blackbodies emit only the fraction $\varepsilon(\lambda)$ of the blackbody radiation, and the remaining fraction, $1-\varepsilon(\lambda)$, is either transmitted, absorbed for opaque objects, or reflected. When the scene is composed of objects and backgrounds of similar temperatures, reflected radiation tends to reduce the available contrast. However, reflections of hotter or colder objects have a significant effect on the appearance of a thermal scene. To illustrate this purpose, a specific sample which is an electrical heater figure 4.a is chosen. The main particularity of the sample is its constant emissivity. In fact the sample is composed of metallic thin layer embedded in a mica matrix. As a consequence, the sample can be warmed up by the Joule effect given by the metallic layers. The second important point is the relative transparency of the mica in the spectral range between 4.5 to $5 \mu \mathrm{m}$. To illustrate that the simultaneous temperature and spectroscopic measurement are possible, a square electrical power was applied to the heater and by using the two images technique (describe before c.f. figure 2) the intensity variation of the sample are recorded. The results of one pixel of the image $(p x=53$ and $p y=88)$ is plotted figure $4 . b$ as function of time. It is clearly show that two regimes occurred, the first at the bottom of the graph where only the proper emission of the sample is measured and a second (top of the graph) where both the proper emission and the spectroscopic contribution are present. Figure 4.c, shows that the measured temperature in proper emission for a given frame can be extracted. To take into account all the reflection and background problems the temperature of the frame number 1 (at room temperature) is also subtracted. This representation allows to recognize the proper thermal field of the sample. It is important to note that the temperature of the metallic plate is higher and quite homogeneous than the mica one, this is because the Joule effect occurs only in the metal. Moreover, thermal gradient is present due to border effect and to the inhomogeneous repartition of dissipated energy from the Joule effect. On the other hand on figure 4.d the spectroscopic field is represented at the same time (or for a given frame). Here it is interesting to see that the intensity is null everywhere excepted where the spectroscopic beam occurred. Moreover due to the high reflecting index of metal at such wavelength, the signal is only transmitted by the mica. The non homogeneous spectrum is due to optical problem already seen for the beam alone depicted by figure 3. 


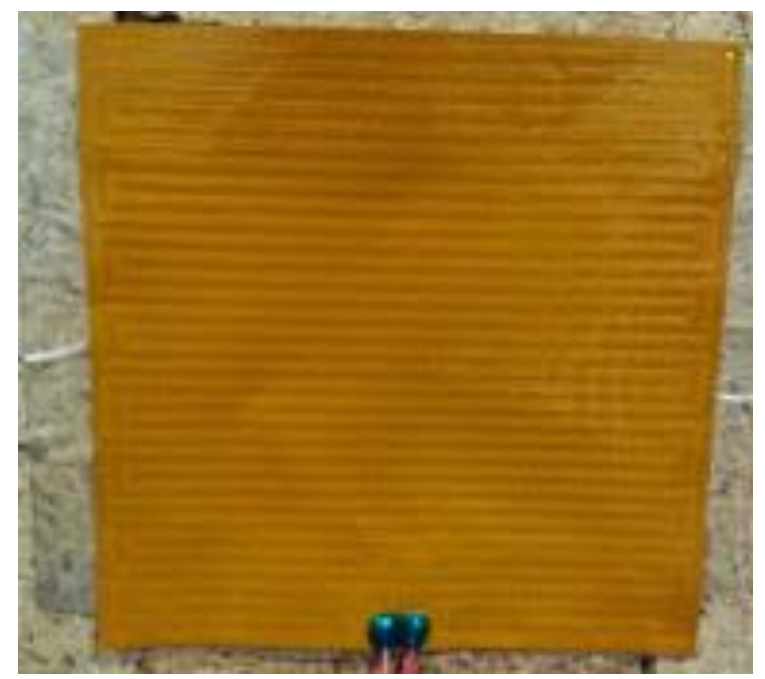

(a)

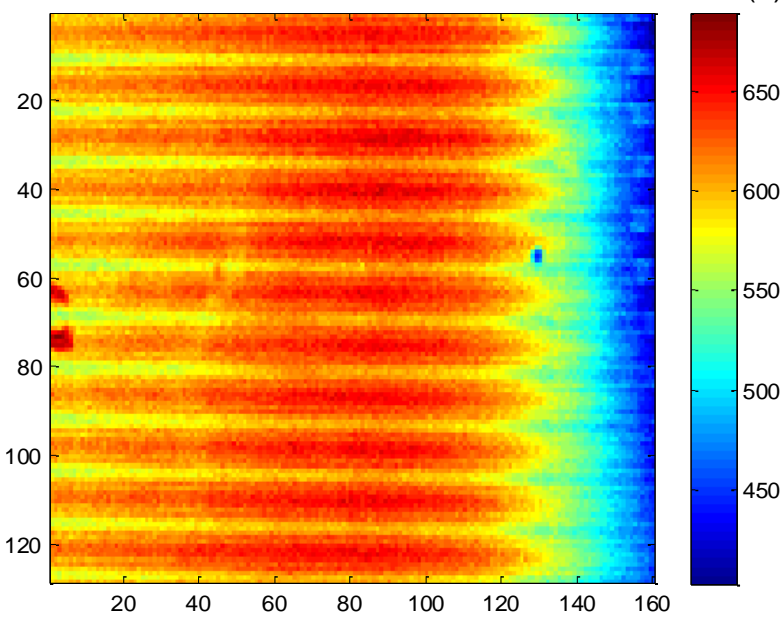



(b)

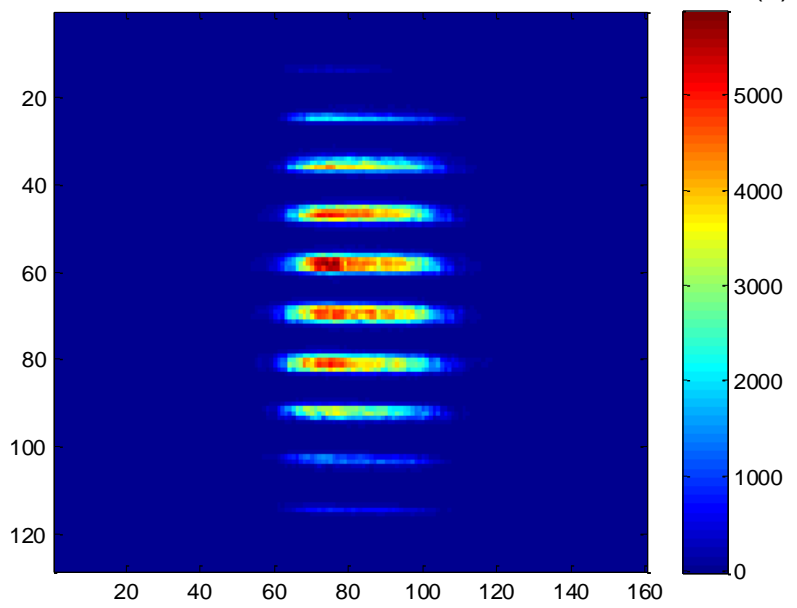

(d)

Fig. 4. a) The chosen sample is composed of metallic thin layer embedded in a mica matrix. b) The simultaneous temperature and spectroscopic measurement are possible, a square electrical power was applied to the heater and by using the two images technique. c) Shows the measured temperature in proper emission for a given frame can be extracted. To take into account all the reflection and background problems the temperature of the frame number 1

(at room temperature) is subtracted. This representation allows to recognize the proper thermal field of the sample. d) The spectroscopic field is represented at the same time (or for a given frame).

More precisely if the spectroscopic field shown on figure 4.c is observed at different wavelength it is possible to detect a intensity variation regarding the nature of the material. Figure 5 sketches the spectroscopic field of the heating resistance at three different wavelengths, it is important to note that the intensity bar located at the right side of each image has different values. In fact for the three observed wavelength the nature of material reveals to have a maximum of absorbance between 4500 and $5000 \mathrm{~nm}$, as illustrated on figure 5.b and c. 


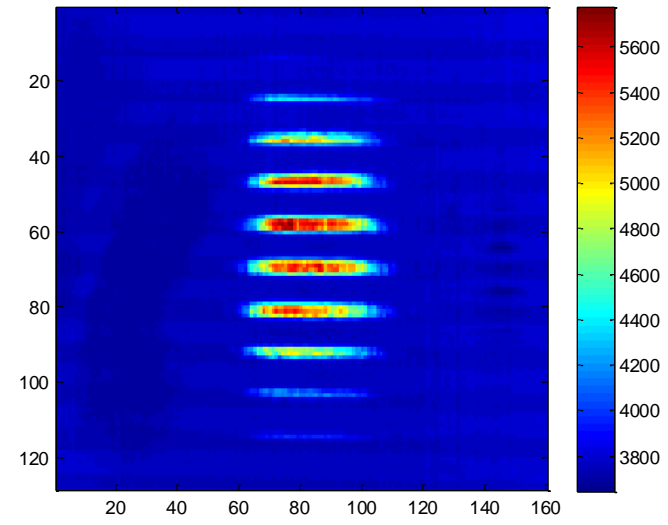

a) $4000 \mathrm{~nm}$



b) $4500 \mathrm{~nm}$

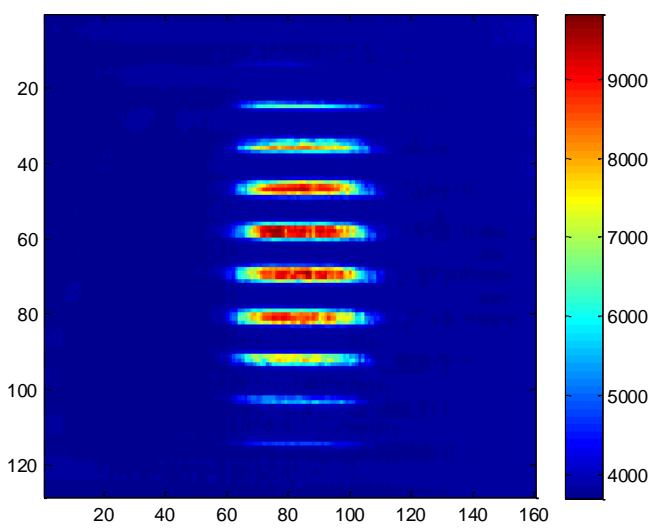

c) $5000 \mathrm{~nm}$

Fig. 5. The spectroscopic field is represented at the same time (or for a given frame) at three different wavelength a) $4000 \mathrm{~nm}$ b) $4500 \mathrm{~nm} \mathrm{C)} 5000 \mathrm{~nm}$

\section{Conclusions and perspectives}

In this paper the simultaneous measurement of IR spectroscopic imaging and temperature fields is presented. A two images processing methodology was proposed to measure both the temperature in proper emission and spectroscopy in transmission. In summary, it has been demonstrated that this spectroscopic imaging and temperature has numerous advantages than conventional spectrometers. It has, however, additional features that IR spectrometers cannot offer, namely, its ability to provide the measurement of the concentration and the temperature at the same time and at different positions with only one image of the sample. Moreover, it is possible to monitor the samples versus the time. In this case, the high spatial resolution and sensitivity of the InSb camera allow to determine the kinetics from data collected by each single pixel in the camera. Thus, with this tool it becomes possible to achieve both thermal analysis (temperature field and thermo physical properties estimation) and chemical analysis (kinetic) over four dimensions: space $(X Y)$, time and wavelength of numerous phenomena determined by this multispectral imaging instrument. 


\section{REFERENCES}

[1] Tran, C. D., Cui, Y., \& Smirnov, S. (1998). Simultaneous multispectral imaging in the visible and near-infrared region: applications in document authentication and determination of chemical inhomogeneity of copolymers. Analytical chemistry, 70(22), 4701-4708.

[2] Sfarra, S., Ibarra-Castanedo, C., Ambrosini, D., Paoletti, D., Bendada, A., \& Maldague, X. (2011). Integrated approach between pulsed thermography, near-infrared reflectography and sandwich holography for wooden panel paintings advanced monitoring. Russian Journal of Nondestructive Testing, 47(4), 284-293.

[3] Ibarra-Castanedo, C., Sfarra, S., Ambrosini, D., et al., Subsurface Defect Characterization in Artworks by Quantitative PPT, QIRT J., 2008, vol. 5, no. 2, pp. 131-149.

[4] Abedin, M. Nurul, et al. The future of single-to multi-band detector technologies review. 2006, Advanced Research Workshop-Future Trends in Microelectronics Up the Nano Creek. 2006.

[5] Pasquini, Celio. "Near infrared spectroscopy: fundamentals, practical aspects and analytical applications." Journal of the Brazilian Chemical Society 14.2 (2003): 198-219.

[6] Reich, Gabriele. "Near-infrared spectroscopy and imaging: basic principles and pharmaceutical applications." Advanced drug delivery reviews 57.8 (2005): 1109-1143.

[7] Lyon, Robbe C., et al. "Near-infrared spectral imaging for quality assurance of pharmaceutical products: analysis of tablets to assess powder blend homogeneity." AAPS PharmSciTech 3.3 (2002): 1-15.

[8] Barbin, Douglas F., et al. "Non-destructive determination of chemical composition in intact and minced pork using near-infrared hyperspectral imaging." Food chemistry 138.2 (2013): 1162-1171.

[9] Rogalski, A., and K. Chrzanowski. "Infrared devices and techniques." Optoelectronics Review 2 (2002): 111136.

[10] Smith, Matthew R., et al. "Hydrated silica on Mars: Combined analysis with near-infrared and thermal-infrared spectroscopy." Icarus 223.2 (2013): 633-648. 